\title{
The effect of the atmospheric refractive index on the radio signal of extensive air showers using Global Data Assimilation System (GDAS)
}

A. Corstanje ${ }^{2}$, P. Mitra ${ }^{1 *}$, A. Bonardi ${ }^{2}$, S. Buitink ${ }^{1}$, H. Falcke ${ }^{2,3,4}$, B. M. Hare ${ }^{5}$, J.R. Hörandel ${ }^{2,3}$, K. Mulrey ${ }^{1}$, A. Nelles ${ }^{2,6}$, J.P. Rachen ${ }^{2}$, L. Rossetto ${ }^{2}$, P. Schellart ${ }^{2,8}$, O. Scholten ${ }^{5,7}$, S. ter Veen ${ }^{2,4}$, S. Thoudam ${ }^{2,9}$, T.N.G. Trinh ${ }^{5}$, T. Winchen ${ }^{1}$

${ }^{1}$ Astrophysical Institute, Vrije Universiteit Brussel, Pleinlaan 2, 1050 Brussels, Belgium

${ }^{2}$ Department of Astrophysics / IMAPP, Radboud University Nijmegen, P.O. Box 9010, 6500 GL, Nijmegen, The Netherlands

${ }^{3}$ NIKHEF, Science Park Amsterdam, 1098 XG Amsterdam, The Netherlands

${ }^{4}$ Netherlands Institute of Radio Astronomy (ASTRON), Postbus 2, 7990 AA Dwingeloo, The Netherlands

${ }^{5}$ KVI-CART, University Groningen, P.O. Box 72, 9700 AB Groningen, The Netherlands

${ }^{6}$ Department of Physics and Astronomy, University of California Irvine, Irvine, CA 92697-4575, USA

${ }^{7}$ Interuniversity Institute for High-Energy, Vrije Universiteit Brussel, Pleinlaan 2, 1050 Brussels, Belgium

${ }^{8}$ Department of Astrophysical Sciences, Princeton University, Princeton, NJ 08544, USA

${ }^{9}$ Department of Physics and Electrical Engineering, Linnéuniversitetet, 35195 Växjö, Sweden

E-mail: pragati.mitra@vub.ac.be

One of the major systematic uncertainties in the measurement of $X_{\max }$ from radio emission of EAS arises from variations of the refractive index in the atmosphere. The refractive index $n$ varies with temperature, humidity and pressure, and the variations can be on the order of $10 \%$ for $(\mathrm{n}-1)$ at a given altitude. The effect of a varying refractive index on $X_{\max }$ measurements is evaluated using CoREAS: a microscopic simulation of the radio emission from the individual particles in the cascade simulated with CORSIKA. We discuss the resulting offsets in $X_{\max }$ for different frequency regimes, and compare them to a simple physical model. Under typical circumstances, the offsets in $X_{\max }$ range from $4-11 \mathrm{~g} / \mathrm{cm}^{2}$ for the $30-80 \mathrm{MHz}$ frequency band. Therefore, for precise measurements it is required to include atmospheric data at the time and place of observation of the air shower into the simulations. The aim is to implement this in the next version of CoREAS/CORSIKA using the Global Data Assimilation System (GDAS), a global atmospheric model based on meteorological measurements and numerical weather predictions. This can then be used to re-evaluate the air shower measurements of the LOFAR radio telescope.

35th International Cosmic Ray Conference - ICRC2017

10-20 July, 2017

Bexco, Busan, Korea

* Speaker. 


\section{Introduction}

In recent years, the field of radio detection of high-energy air showers has advanced quite rapidly. Estimating the depth of shower maximum $X_{\max }$ with improved accuracy is of great interest for the study of primary particle composition [1]. The highest precision to the determination of $\mathrm{X}_{\max }$ with radio technique is currently achieved with the LOFAR radio telescope, situated in the north of the Netherlands. The central ring of LOFAR consists of 288 low-band antennas within the diameter of 320 meters, recording cosmic ray events within 30-80 MHz. Additionally, there are also 288 high-band antennas measuring in the 110-190 MHz range [2]. The densely instrumented core of LOFAR gives the opportunity to investigate the radio footprint i.e. the lateral intensity distribution in close detail and enables to infer $X_{\max }$ to a precision of $20 \mathrm{~g} / \mathrm{cm}^{2}$ which is comparable to the precision achieved influorescence detectors [3].

The measured lateral intensity distributions are compared to CoREAS [4], a simulation package of the radio emission from the individual particles in the cascade simulated with CORSIKA [5]. The detected radio signal depends on the difference in travel times of radio waves and particles. Therefore, it is important to apply an accurate value of the refractive index $\mathrm{n}$. Variations in the refractive index lead to changes in the radio intensity footprint on the ground because the angle of peak emission depends on n [6]. In CORSIKA, the standard US atmosphere is used as the default model atmosphere featuring constant temperature lapse with altitude and zero humidity. Thus a realistic model calls for more accurate description of atmosphere.

The Global Data Assimilation System (GDAS) developed at NOAA's National Centers for Environmental Prediction (NCEP) is a tool to study realistic atmosphere [7]. It is run four times a day $(0,6,12$, and 18 UTC) and provides a 3-, 6- and 9-hour forecast based on the interpolation of the meteorological measurements from all over the world including weather stations on land, ships, and airplanes as well as radiosondes and weather satellites. The three hourly data are available at 23 constant pressure levels, from $1000 \mathrm{hPa}$ (roughly sea level) to $20 \mathrm{hPa}(\approx 26 \mathrm{~km}$ ) on a global $1^{\circ}$ spaced latitude-longitude grid $\left(180^{\circ}\right.$ by $\left.360^{\circ}\right)$. Each data set is complemented by data for the surface level. The data are stored in weekly files and made available online.

\section{Analysis and results}

The effect of refractivity on the radio footprint can be better comprehended from a simple toy model as depicted on the left panel of Fig 1. Because of propagation effects, the radio pulse is compressed, depending on the viewing angle. The compression is maximal at the Cherenkov angle where the emission is thus coherent up to much higher frequencies. Roughly above $100 \mathrm{MHz}$, the emission strongly peaks at the Cherenkov angle, forming a ring of radiation on the ground [8]. Below $100 \mathrm{MHz}$, the radiation pattern has a more complicated structure, but its size still scales with the Cherenkov angle. The relation between $\mathrm{n}$ and Cherenkov angle $\alpha$ is given by

$$
\cos \alpha=\frac{1}{\beta n(h)} .
$$

The model is based on the assumption that most of the radiation is produced near $\mathrm{X}_{\max }$ and the size of the footprint scales with distance to $X_{\max }$ and with the Cherenkov angle at that distance. if the 
refractive index is higher than expected, the Cherenkov angle becomes larger. The measured size of the radiation footprint now corresponds to a lower emission altitude, and therefore a larger $X_{\max }$. Using CoREAS simulations we have also determined the systematic offset in $\mathrm{X}_{\max }$ measurement for a $10 \%$ increase in refractivity. Two ensembles of showers are compared, by taking one shower from the ensemble of higher refractivity and fitting the lateral intensity distribution by all 50 from normal refractivity ensemble. A mean squared error is used as a fit quality measure. For every shower, fit quality is plotted against $X_{\max }$ showing a minimum with a quadratic dependence and therefore fitted to a parabola. The location of the minimum of the parabola is used as an estimator for $X_{\max }$. An typical example is shown on the right panel of Fig 1. The average between the difference of the newly fitted and true $X_{\max }$ yields the systematic offset in $X_{\max }$. Details of this analysis can be found in [6]. With a linear fit to the points the offsets found are nearly half of that obtained from Cherenkov toy model described above between $30-80 \mathrm{MHz}$ as shown in Fig 2. This indicates that Cherenkov time compression is not the only determining factor behind the shape of the radio footprint. The offset ranges from $9 \mathrm{~g} / \mathrm{cm}^{2}$ for $15^{\circ}$ zenith to $18 \mathrm{~g} / \mathrm{cm}^{2}$ for $45^{\circ}$ zenith. The relation for the offset $\Delta X$ as a function of distance to $X_{\max }(R)$ for $10 \%$ higher refractivity is given as

$$
\Delta X_{10 \%}=3 \frac{R}{1 \mathrm{~km}}-1.37 \mathrm{~g} / \mathrm{cm}^{2} .
$$

and for higher frequencies the difference in offset between simulation and Cherenkov model decreases because the the toy model more accurately describes the radiation pattern [6]. In order to
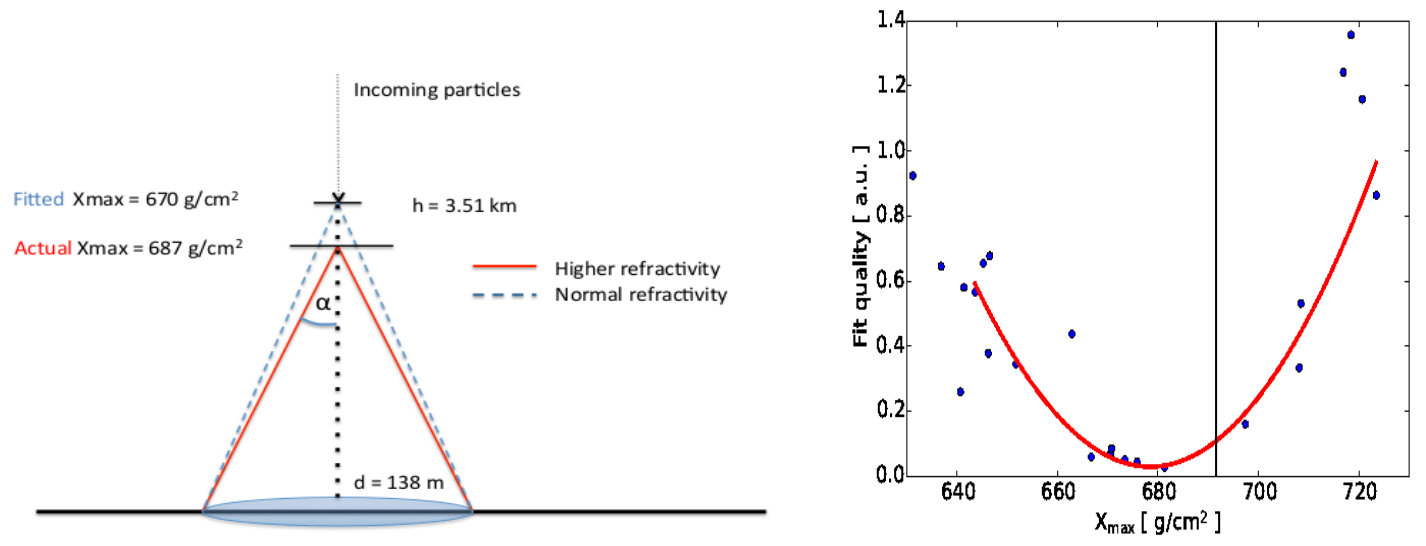

Figure 1: Left- Sketch of Cherenkov model: the effect of an increase in $n$ is shown schematically, for a proton primary particle of $10^{17} \mathrm{eV}$ from zenith having an average $X_{\max }=670 \mathrm{~g} / \mathrm{cm}^{2}$ as obtained from CoREAS simulations, corresponding to an altitude of $3.51 \mathrm{~km}$. $10 \%$ higher refractivity than the default value used in CoREAS ( $N=292$ at sea level), results in a higher $X_{\max }$ (red line), than the normal one (blue line). The modeled difference in $X_{\max }$ amounts to $17 \mathrm{~g} / \mathrm{cm}^{2}$ [6]. Right- Fit quality as a function of $X_{\max }$. The simulated $X_{\max }$ for the shower with higher refractivity is indicated by the black vertical line. The minimum gives the best fit value. The scatter around the minimum arises due to shower to shower fluctuation.

model a realistic atmosphere one needs to obtain the suitable atmospheric parameters from GDAS. Parameters like temperature, relative humidity and pressure can be directly extracted from GDAS files. Other parameters can be calculated using physical relations involving those first three pa- 


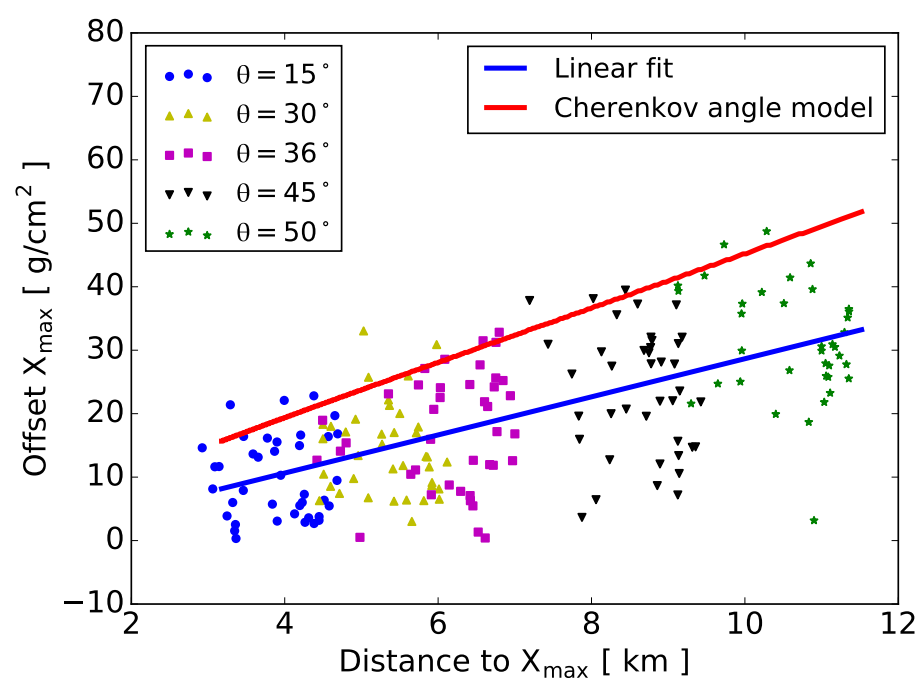

Figure 2: $\mathrm{X}_{\max }$ offset for different zenith angles for LOFAR low band frequency range (30-80 MHz), where the points represent individual simulated shower. The blue line is a linear fit to the data and the red line is from the Cherenkov model [6].

rameters. To calculate the air density, the relative humidity must be converted into water vapor pressure, the partial pressure of water in air in $\mathrm{hPa}$. This conversion depends on air temperature. The following approximation of the empirical Magnus formula is used in the calculations [9]:

$$
e=\frac{h}{100 \%} \times 6.1070 \times \exp \left(\frac{21.88 t}{265.5+t}\right) \quad \text { for } t \geq 0^{\circ} \mathrm{C}
$$

and

$$
e=\frac{h}{100 \%} \times 6.1070 \times \exp \left(\frac{17.15 t}{234.9+t}\right) \quad \text { for } t \leq 0^{\circ} \mathrm{C}
$$

The density in can be calculated from ideal gas equation as

$$
\rho=\frac{P M_{\text {air }}}{R T}
$$

where $P, T$ and $R$ are pressure, temperature, universal gas constant respectively and $M_{\text {air }}$ is the molar mass of air which can be calculated from the contributions of dry air, water vapor and $\mathrm{CO}_{2}$. Next, the refractivity $\mathrm{N}$ as a function of humidity, pressure and temperature can be expressed as

$$
N=77.6890 \frac{p_{d}}{T}+71.2952 \frac{p_{w}}{T}+375463 \frac{p_{w}}{T^{2}}
$$

with $p_{w}, p_{d}$ and $T$ being the partial water vapor pressure (hPa), partial dry air pressure (hpa) and temperature respectively. In the regime of radio frequency higher value of humidity increases the refractivity. On the right panel of Fig $\mathbf{3}$ it is shown that the day to day humidity has a huge variance. It is thus neccessary to use the detailed atmospheric conditions at the time of observation.

In order to incorporate atmospheric parameters extracted from GDAS to CORSIKA/CoREAS 

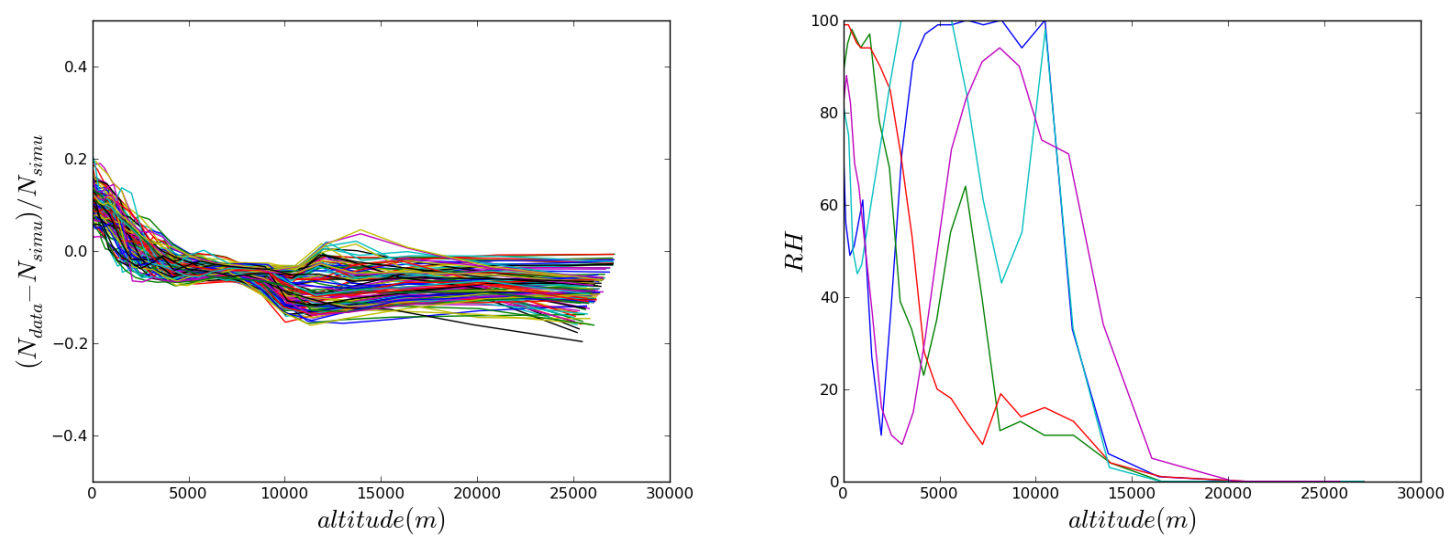

Figure 3: Left- The relative refractivity plot as a function of altitude for different times over 3 years for 100 cosmic ray events recorded at Lofar. Relative refractivity is defined as the ratio of difference between data and simulation to data. It fluctuates from approximately $20 \%$ above default value to $10 \%$ below the default value used in CoREAS between 0-5 km of altitude. This variation is indeed is the effect of rapidly varying temperature and humidity between the above mentioned altitude range. Also between 3-8 km the relative variation in refractivity is about $3 \%-5 \%$. This altitude range is of interest as this is usually where the shower reaches its maximum. Right- Relative humidity plot as a function of altitude for 5 random events. It changes quite rapidly over the altitude and becomes negligible after around $12 \mathrm{~km}$ as this marks the end of troposphere.

we have developed a script that will download the required GDAS file given the time and location of observation of the event and return values of refractive index between ground and highest GDAS level within $1 \mathrm{~m}$ interval. It also fits the density profile according to the standard 5 layered atmospheric model used in CORSIKA[5]. In this model the density $\rho(h)$ follows an exponential dependence on the altitude leading to the functional form of mass overburden $T(h)$ which is the density integrated over height as

$$
T(h)=a_{i}+b_{i} \cdot e^{-h / c_{i}} \quad i=1, \ldots, 4
$$

Thus the density

$$
\rho(h)=b_{i} / c_{i} \cdot e^{-h / c_{i}} \quad i=1, \ldots, 4
$$

In the fifth layer the overburden is assumed to decrease linearly with height. The parameters $a_{i}, b_{i}$ and $c_{i}$ are to be obtained in a manner that the function $T(h)$ is continuous at the layer boundaries and can be differentiated continuously. We distribute 24 GDAS density points into 3 layers with the layers having boundaries approximately around $4 \mathrm{~km}$ for first layer, $9.5 \mathrm{~km}$ for second layer and between $26-28 \mathrm{~km}$ for the third layer. A point to note here is that the layer boundaries are not constant but rather can change slightly depending on the place of observations and time. Next, we fit the data to Eq 2.8 in the following way-

For layer 1 we fit the density profile with two free parameters. Then we calculate density $\rho_{1}$ at boundary 1 from Eq 2.8 using the obtained parameters $b_{1}, c_{1}$. The boundary condition that the density has to be continuous at the boundaries reduces the number of free parameters to 1 and the 
parameter $b_{2}$ for second layer can be expressed as a function of $\rho_{1}$ and $c_{2}$ with $c_{2}$ being the only free parameter. Similarly we continue the same procedure for the higher layers. Since now we have the smooth profile for density we can analytically solve for the parameter $a$ in Eq 2.7 exploiting the boundary conditions for mass overburden. The parameterization for fifth layer is adapted from the US standard atmosphere [5]. An example of the fit is shown in Fig 4.
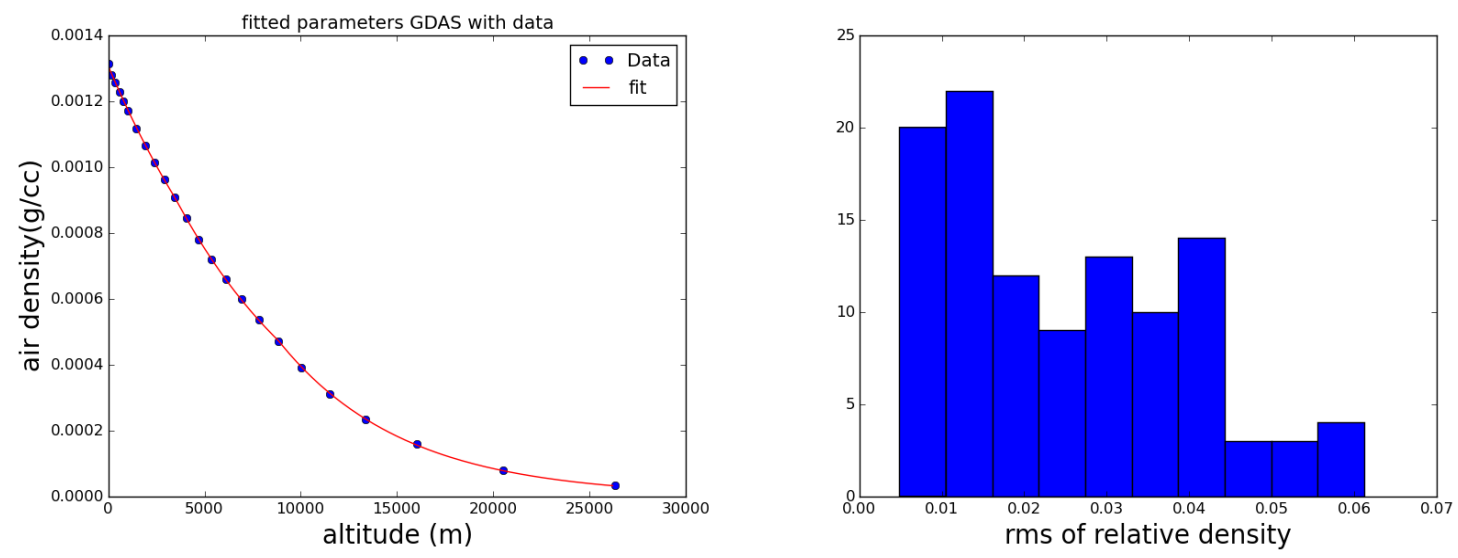

Figure 4: Left- density vs altitude profile for an arbitrary event, blue dots are GDAS data points and the red line is the fitted profile using the CORSIKA parameterization. Right-Histogram for rms value of relative density for 100 different events. The relative density is defined as $\frac{\rho_{\text {fit }}-\rho_{\text {data }}}{\rho_{\text {fit }}}$. The small value indicates the good quality of fit though a small bias towards positive values is introduced probably because of the imposed boundary conditions.

\section{Conclusion and outlook}

For mass composition analysis the accuracy of the $X_{\max }$ measurement is crucial. For $X_{\max }$ measurements with the radio technique we have investigated the systematic error due to variations in the atmospheric refractive index inparticular for LOFAR measurements; for higher refractivity the offsets are found to be proportional to the distance to $X_{\max }$ for different zenith angles. We also have proposed a toy model based on the Cherenkov emission at $X_{\max }$ altitude that qualitatively describes this effect especially for higher frequency range $(120-250 \mathrm{MHz})$. Given variations in $N$ on the order of $4 \%$, from the atmospheric effects the offsets would range from about 3.5-11 $\mathrm{g} / \mathrm{cm}^{2}$ for $30-80 \mathrm{MHz}$, although one can not use a single value of refractivity in simulation as it does not provide full information of realistic atmosphere. Using GDAS we studied the local atmospheric profiles for refractivity and humidity at the time of several cosmic ray events recorded at LOFAR. The parameters show significant variations over time. The relative variation in refractivity is found to be around 3\%-5\% between $3-8 \mathrm{~km}$ of altitude. We have implemented GDAS atmospheric profiles with local refractive index lookup table and improved density parametrization to COREAS/CORSIKA. These modifications will be included in a future release of CORSIKA/CoREAS together with a standalone script that downloads GDAS files and produces atmosphere profiles and CORSIKA steering files. The script is flexible and is able to create profiles for any location on Earth. However, for very specialized applications, such as near-horizontal showers, 
further development will be needed. Also current work is going on the re-evaluation of LOFAR measurements with improved atmosphere.

\section{Acknowledgement}

The LOFAR cosmic ray key science project acknowledges funding from an Advanced Grant of the European Research Council (FP/2007-2013) / ERC Grant Agreement no 227610. The project has also received funding from the European Research Council (ERC) under the European Union's Horizon 2020 research and innovation program (grant agreement No 640130). We furthermore acknowledge financial support from FOM, (FOM-project 12PR304) and NWO (VENI grant 639041-130). AN is supported by the DFG (research fellowship NE 2031/1-1). LOFAR, the Low Frequency Array designed and constructed by ASTRON, has facilities in several countries, that are owned by various parties (each with their own funding sources), and that are collectively operated by the International LOFAR Telescope foundation under a joint scientific policy.

\section{References}

[1] S. Buitink et al. A large light-mass component of cosmic rays at $10^{17}-10^{17.5} \mathrm{eV}$ from radio observations. Nature, 531:70, 2016.

[2] P. Schellart et al. Detecting cosmic rays with the LOFAR radio telescope. Astronomy and Astrophysics, 560(A98), 2013.

[3] S. Buitink et al. Method for high precision reconstruction of air shower $\mathrm{X}_{\mathrm{max}}$ using two-dimensional radio intensity profiles. Phys. Rev. D, 90(8), 2014.

[4] Tim Huege and Clancy W. James. Full Monte Carlo simulations of radio emission from extensive air showers with CoREAS. In Proceedings, 33rd International Cosmic Ray Conference (ICRC2013): Rio de Janeiro, Brazil, July 2-9, 2013, page 0548, 2013.

[5] D. Heck et al. CORSIKA: A Monte Carlo code to simulate extensive air showers. Report FZKA, 6019, 1998.

[6] A. Corstanje et al. The effect of the atmospheric refractive index on the radio signal of extensive air showers. Astropart. Phys., 89:23-29, 2017.

[7] National oceanic and atmospheric administration, global data assimilation system.

[8] A. Nelles et al. Measuring a Cherenkov ring in the radio emission from air showers at 110 âĂŞ190 MHz with LOFAR. Astropart. Phys., 65:11-21, 2015.

[9] P. Abreu et al. Description of Atmospheric Conditions at the Pierre Auger Observatory using the Global Data Assimilation System (GDAS). Astropart. Phys., 35:591-607, 2012. 